\title{
Effect of Helicobacter pylori infection on pregnancy rates and early pregnancy loss after intracytoplasmic sperm injection
}

This article was published in the following Dove Press journal:

International Journal of Women's Health

4 October 2011

Number of times this article has been viewed

Masomeh Hajishafiha'

Mohammad Ghasemi-rad'

Aishe Memari'

Siamak Naji'

Nikol Mladkova ${ }^{2}$

Vida Saeedi'

'Urmia University of Medical Sciences, Urmia, Iran; ${ }^{2}$ Institute of Cell and

Molecular Science, London, UK
Correspondence: Masomeh Hajishafiha Urmia University of Medical Sciences, Resalat Boulevard, Jahad Avenue, Urmia, Iran 57|47-83734

Tel +98 44I 235356 I

Mob +989143485284

Fax $+9844|235356|$

Email mhajshafiha@gmail.com
Background: There is a need to elucidate what affects the implantation and early pregnancy course in pregnancies conceived with assisted reproductive technology (ART) so that pregnancy rates and outcomes can be improved. Our aim was to determine the role of maternal Helicobacter pylori infection.

Material and methods: We did a prospective study of 187 infertile couples undergoing intracytoplasmic sperm injection (ICSI) and segregated those according to underlying infertility etiology. We assessed the status of $H$. pylori IgG antibodies and anti-CagA IgG antibodies by ELISA assay. All pregnancies were followed for early pregnancy loss (EPL, first 12 weeks).

Results: The likelihood of $H$. pylori infection increased with age (1.01,95\% confidence interval $[\mathrm{CI}]: 1.0-1.13 ; P=0.040)$ but there was no association with EPL. Women infected with CagApositive strains were more likely to have EPL $(19.39,95 \%$ CI: $1.8-208.4 ; P=0.014)$. Women with tubal factor or ovulatory disorder infertility were more likely to abort early $(12.95,95 \%$ CI: $1.28-131.11 ; P=0.030,10.84,95 \%$ CI: $1.47-80.03 ; P=0.020$, respectively). There was no association between EPL and age, number of embryos formed or transferred, or number of oocytes retrieved.

Conclusion: Our findings suggest that infection with CagA-positive H. pylori strains is linked to an increase in women's potential to abort early (possibly through increased release of inflammatory cytokines). In addition, tubal factor and ovulatory disorder infertility are linked to EPL after ICSI due to unknown mechanisms. Proposals to eradicate $H$. pylori infection prior to ICSI could lead to a decrease in EPL after ART.

Keywords: Helicobacter pylori, early pregnancy loss, early abortion, infertility, intracytoplasmic sperm injection, CagA

\section{Introduction}

Infertility is defined as the failure to conceive after 1 year of regular, unprotected intercourse. ${ }^{1}$ This condition affects up to one in seven couples in industrialized countries, ${ }^{2}$ and it is one of the most prevalent chronic health disorders involving young adults. ${ }^{3}$

Several methods have been introduced to help infertile couples conceive, including intracytoplasmic sperm injection (ICSI). ICSI was introduced in 1992 as a solution for male infertility ${ }^{4}$ and in 2007 , ICSI represented $48 \%$ of all in vitro fertilization treatments in the UK. ${ }^{5}$ In this procedure, a single sperm is injected directly into the oocyte cytoplasm, and it represents a very effective therapy option in men with very low sperm counts. The clinical pregnancy rates after ICSI have been reported to reach $39.2 \%$ per embryo transfer, ${ }^{6}$ and the overall success of ICSI seems dependent on factors such as sperm retrieval method, ${ }^{7}$ maternal age, and whether the infertility is primary or secondary. ${ }^{8}$ In addition, miscarriage significantly reduces the initial success of assisted reproduction treatment. ${ }^{9}$ 
Early pregnancy loss (EPL) after ICSI has been linked to obesity and increased maternal age. ${ }^{10}$ However, additional unknown barriers affect the efficiency of ICSI treatment and require further research.

Helicobacter pylori infection affects more than half of the world's population, ${ }^{11}$ and is most commonly associated with chronic gastritis, which subsequently increases the risk of many serious complications, including gastric cancer. ${ }^{12}$ Additionally, there are limited reports indicating potential correlation between infertility and $H$. pylori infection. ${ }^{13,14}$ It has been shown in mice that $H$. pylori infected mice show a decrease in implantation rates, and their offspring are of low birth weight. ${ }^{15}$ In infertile human males, $H$. pylori infection was shown to be associated with low sperm quality compared to uninfected patients. ${ }^{14}$

The pathogenicity of an H. pylori isolate depends on strain-specific factors. ${ }^{16}$ The cag pathogenicity island, for which cytotoxin-associated gene A (CagA) is the marker, has been associated with both duodenal ulcer and gastric cancer, ${ }^{17}$ and infection with CagA-positive strain is generally associated with a higher level of inflammatory mediators compared to CagA-negative strains.

In CagA-positive male patients, a significant reduction in sperm motility was observed along with increased apoptosis and necrosis ${ }^{14}$ but no studies describing potential role of maternal $H$. pylori infection in pregnancy course exist. Should the detrimental effect of $H$. pylori on pregnancies conceived by assisted reproductive technology (ART) be confirmed, appropriate treatment of $H$. pylori infection prior to ART treatment could improve the treatment success.

Our aim was to investigate the link between maternal H. pylori infection and implantation rates and EPL in patients undergoing ICSI.

\section{Methods}

\section{Participants}

Between January 2009 and September 2009, we did a prospective cohort study, to which we consecutively enrolled women undergoing ICSI at the Centre for Treatment and Research in Infertility of Shahid Mottahari hospital in Urmia, Iran. The Centre for Treatment and Research in Infertility is the only infertility clinic in the West Azarbaijan province of Iran, and all infertile patients in the area are referred to this centre. All patients were of similar age and lived in the same region. Patients' infertility was defined as a failure to conceive after 1 year of unprotected intercourse.

The study was approved by the university Institutional Review Board and Ethics Committee. Informed written consent was obtained from all participants in the presence of a witness. The study is summarized in a flowchart (Figure 1).

\section{Procedures}

We treated women undergoing ICSI with standard clinical and laboratory protocols.

Briefly, couples initially underwent routine fertility work-up consisting of semen analysis, sonohysterography and female hormone testing (luteinizing hormone [LH], follicle stimulating hormone [FSH], prolactin, thyroid stimulating hormone [TSH], estradiol). Hormone levels were evaluated on the third day of the patient's menstrual cycle.

Ovarian stimulation with gonadotropin-releasing hormone (GnRH) agonist buserelin acetate (Suprefact ${ }^{\circledR}$; Hoechst AG, Frankfurt, Germany), at a dose between $0.3-0.5 \mathrm{mg} / \mathrm{SC}$, was started during the midluteal phase in a long protocol manner starting on the 21 st day of the previous cycle. Then a daily injection of human menopausal gonadotropin (HMG) (Menogon $^{\circledR}$, Ferring, Kiel, Germany) was administered and the GnRH dose was lowered to $50 \%$ upon the administration of HMG.

The dose of HMG was increased in accordance with individual patient's response and in accordance with a series of transvaginal sonographic observations until at least two follicles reached a mean diameter of $18 \mathrm{~mm}$. Then 5000-10000 IU of HCG (Pregnyl ${ }^{\circledR}$, Merck/Schering-Plough Pharmaceuticals Inc, Sumneytown Pike, North Wales) was injected intramuscularly and after 34-36 hours, transvaginal ultrasound-guided oocyte retrieval was performed.

\section{Spermatocyte preparation}

Ejaculates were obtained immediately before or shortly after oocyte retrieval.

In cases of obstructive and non-obstructive azoospermia, spermatozoa were obtained by microsurgical epididymal sperm aspiration (MESA) or testicular sperm extraction (TESE), respectively.

\section{Intracytoplasmic sperm injection}

Oocytes were microscopically examined for the presence of cytoplasmic abnormalities before sperm injection. ICSI treatment was performed 5-8 hours after oocyte retrieval.

The technique of ICSI has previously been described in detail. ${ }^{18}$ In brief, at a magnification of $\times 400$, a single spermatozoon was immobilized by mechanical damage to its tail. Prior to ICSI, the oocyte was held in place with a holding pipette (Eppendorf, Hamburg, Germany) at 9 O'clock. The 


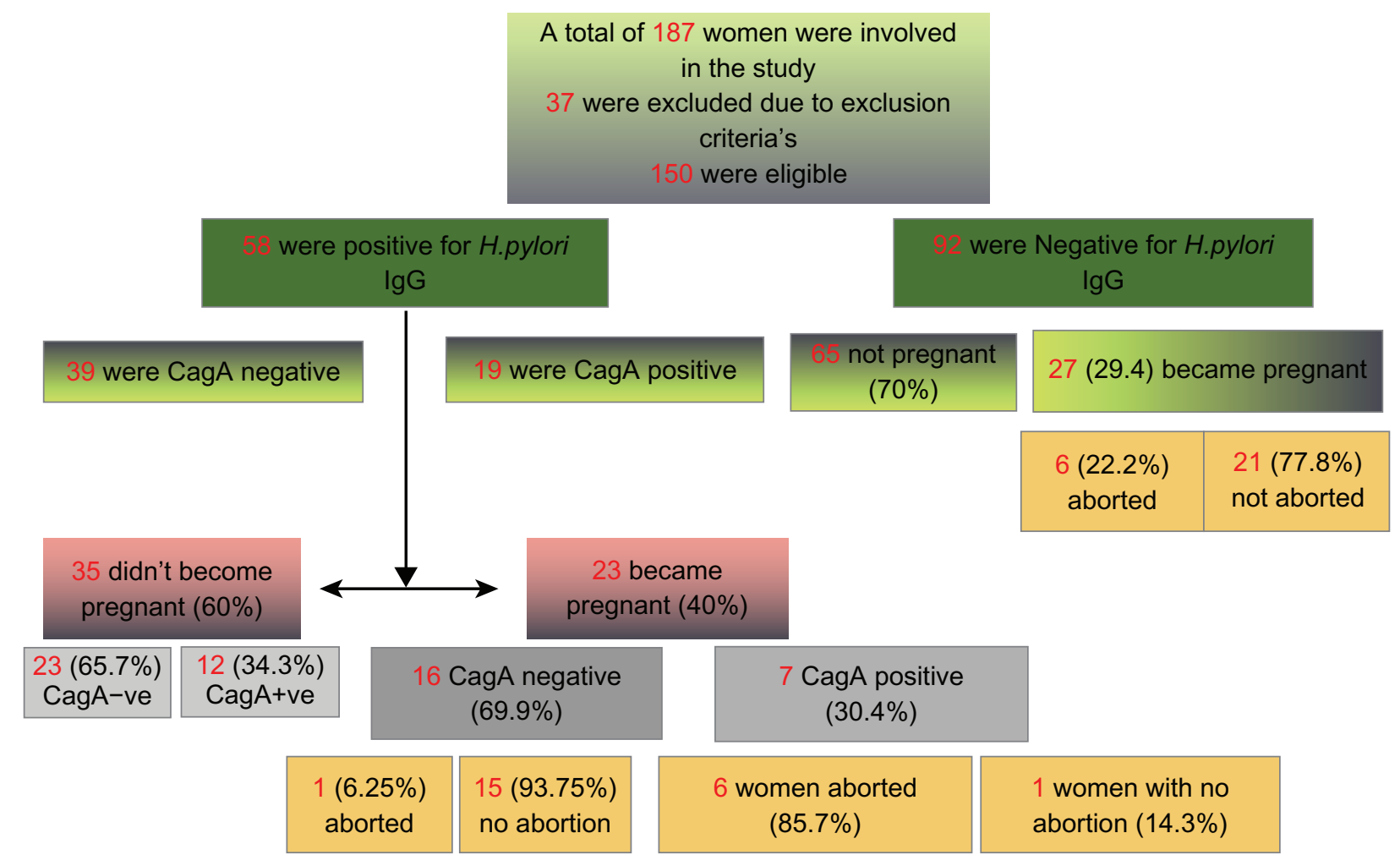

Figure I Flowchart summarizing the study.

first polar body was located on the 6 O'clock position. After penetrating both the zona pellucida and the oolemma, a small volume of the oocyte cytoplasm was aspirated to activate the egg. The previously immobilized spermatozoon was placed near the horizontal axis. Withdrawal was conducted carefully to prevent leakage from the oocyte. One embryologist performed all ICSI treatments.

After sperm injection, fertilized oocytes were placed in individual culture media dishes in an incubator (Labotech, Berlin, Germany), using an environment with $5 \% \mathrm{CO}_{2}$, at $37^{\circ} \mathrm{C}$, in a filtered humidified atmosphere. Normal fertilization and survival was assessed at 14-18 hours after sperm injection.

If at least one embryo of grade $\mathrm{A}$ to $\mathrm{C}$ was identified 48-72 hours after ovarian puncture, it was transferred into the uterine cavity.

\section{Embryo grading}

Prior to the transfer procedure (approximately 48 hours post-insemination), the embryo development and morphology were determined, and the embryos for transfer were selected. Each embryo was assessed according to the blastomere number, the degree of fragmentation, the uniformity of blastomeres, and the presence of multinucleated blastomeres.

\section{Intracytoplasmic sperm injection (ICSI)}

Grade A were embryos with equally sized blastomeres, cells without granulation, and with regular zona pellucida. Grade B were those with equally divided cells and $\leq 10 \%$ granulation. Grade C were those with unequal cells and $20 \%$ granulation. Embryos with unequally-sized blastomeres or $>20 \%$ fragments, unequally-sized blastomeres or $>50 \%$ fragments, and $>80 \%$ fragmentation or with no detectable blastomeres were not transferred.

\section{Patient follow-up after embryo transfer}

Patients received $30 \mathrm{mg}$ of intramuscular progesterone twice-a-day (progesterone $50 \mathrm{mg} / \mathrm{IM}$, Aburaihan Co, Tehran, Iran) after embryo transfer, to reinforce the luteal phase. Serum human chorionic gonadotropin ( $\mathrm{hCG}$ ) was measured 14-15 days post-transfer.

In those with a positive hCG response, we measured $\mathrm{hCG}$ again after 2 days. Patients were followed for 12 weeks sonographically to determine early abortion (week 4, 6, 9, 12).

\section{Determination of antibodies against $H$. pylori}

Complete blood count $(\mathrm{CBC})$ is an integral part of the routine check-up prior to surgery for all patients undergoing ICSI. Upon drawing blood for $\mathrm{CBC}$, an additional $2 \mathrm{~mL}$ of patients' 
blood were taken for $H$. pylori IgG assessment. The blood was centrifuged and the serum was kept at $-20^{\circ} \mathrm{C}$ until the day of examination.

The status of $H$. pylori IgG was determined serologically using enzyme-linked immunosorbent assay (ELISA) (Serum Anti-H. pylori IgG, H. pylori CagA-Ig, Diaplus Inc, North York, ON). Titer levels $>20$ for IgG and $>5$ for CagA were considered positive. If a patient was positive for anti-H.pylori $\mathrm{IgG}$, she also underwent further assessment to determine the anti-CagA IgG status.

\section{Statistical analysis}

Statistical analysis was performed using SPSS (v 16; IBM, Chicago, IL) and R package (http://www.r-project.org). We used a logistic regression to predict EPL from age, H. pylori status, CagA positivity, underlying infertility etiology, number of oocytes obtained, number of embryos formed, and number of transferred embryos. A $P$-value less than or equal to 0.05 was considered statistically significant.

\section{Results}

We enrolled 187 and assessed 150 women undergoing ICSI for pregnancy rates, $H$. pylori seropositivity, and EPL. The mean age of participating women was 30.6 years (SD 5.8). The mean age of women who conceived after ICSI and of women who were unable to conceive after ICSI was significantly different $(P<0.0001$, unpaired $t$-test $)$.

Seventeen couples (11\%) had secondary infertility and $133(89 \%)$ primary infertility. The primary diagnosis of infertility was male factors: oligospermia (sperm count $<5$ million); asthenospermia (forward sperm motility $<5 \%$ ); teratospermia (normal sperm morphology $<4 \%$ ); obstructive azoospermia and non-obstructive azoospermia; retrograde ejaculation; and immunologic factors in 81 (54\%) couples, followed by tubal factor (laparoscopically diagnosed bilateral tubal obstruction) in 11 (7\%); ovulation disorders (patients not responding to ovulation stimulation, intrauterine insemination (IUI), and with high FSH levels) in 17 (11\%); and idiopathic infertility in 41 (27\%) (Figure 2). In women receiving embryos, 47 (31\%) had one embryo transferred, $40(27 \%)$ had two embryos transferred, 48 (32\%) had three transferred, eight $(5.3 \%)$ had four transferred and seven $(4.6 \%)$ had five or more transferred.

We excluded from the study and final analysis (Table 1) women with unilateral or bilateral hydrosalpinx $(n=1)$, ovarian cyst formation following the $\mathrm{GnRH}$ administration diagnosed by TVS on the 2nd day of the menstrual cycle

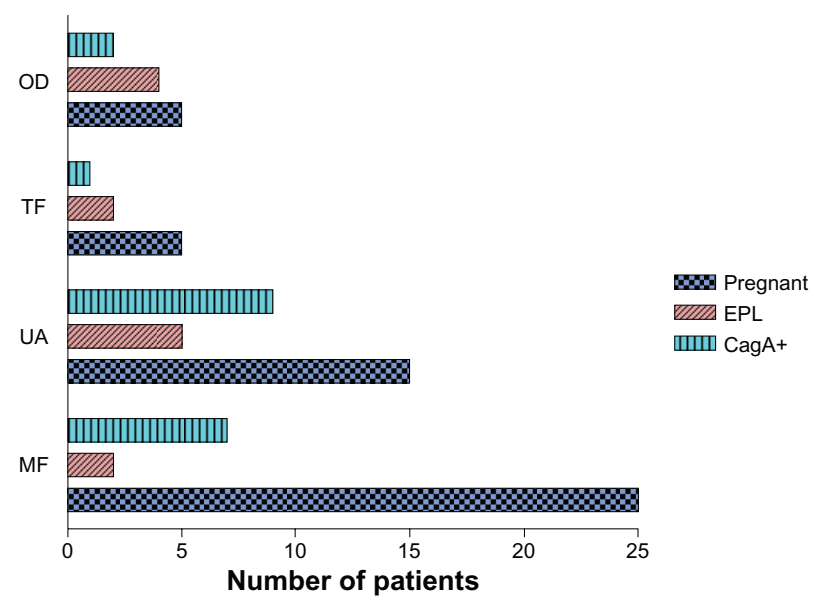

Figure 2 Pregnancy rates, EPL, and infection with CagA-positive strains distributed across groups with different infertility etiology.

Abbreviations: MF, male factor; UA, unknown etiology; TF, tubal factor; OD, ovulatory dysfunction.

$(n=2)$, history of pregnancy loss $(n=2)$, ectopic pregnancy following ICSI $(n=4)$, endometriosis $(n=3)$, patients who in the previous 3 months took medications that might lead to false negative results (eg, tetracycline and omeprazole, $\mathrm{n}=4$ ). We also excluded patients with no follicles or less than two follicles formed after ovarian stimulation $(n=3)$, and with one or no oocyte obtained at follicle puncture $(n=5)$.

Fifty-eight patients (39\%) were positive for IgG antibodies against $H$. pylori, while 92 patients $(61 \%)$ were negative. Nineteen patients $(13 \%)$ with $\operatorname{IgG}$ against $H$. pylori were also positive for CagA. Using a linear model, we noted a significant association between IgG positivity and maternal age $(1.01,95 \%$ confidence interval $[\mathrm{CI}]: 1.0-1.13 ; P=0.040$; but not CagA positivity; $P=0.41)$.

We noted a significant association between CagA positivity and EPL (19.39, 95\% CI: $1.8-208.4 ; P=0.014)$.

There was a significant association between EPL and underlying infertility etiology (12.95, 95\% CI: 1.28-131.11, $P=0.03$ for tubal factor, $10.84,95 \%$ CI: $1.47-80.03, P=0.02$ for ovulation disorder).

Out of 58 patients with $H$. pylori infection, 23 became pregnant after ICSI, and among $92 \mathrm{H}$. pylori negative patients, 27 became pregnant (1.58, 95\% CI: 0.79-3.16; $P=0.19)$. This indicates that $H$. pylori infection does not affect pregnancy rates after ICSI.

Among $23 \mathrm{H}$. pylori positive pregnant women, seven (30\%) were also positive for CagA strains, and among $35 \mathrm{H}$. pylori positive women that did not conceive after ICSI, 12 (34\%) were also positive for CagA. There was no significant link between CagA infection and pregnancy following ICSI $(0.83,95 \%$ CI: $0.26-2.56 ; P=0.76)$. 
Table I Early pregnancy loss related to maternal age, $H$. pylori infection, CagA positivity, underlying infertility etiology (IA), number of oocytes retrieved (OR), number of embryos formed (EF), and number of embryos transferred (ET)

\begin{tabular}{llllll}
\hline & Odds ratio & Lower $\mathbf{C l}$ & Upper Cl & P value & Number \\
\hline Age & 0 & 0.79 & 1.04 & 0.15 & 150 \\
IgG positivity & 0.47 & 0.05 & 4.52 & 0.52 & 58 \\
CagA positivity & 19.39 & 1.8 & 208.4 & $0.01 *$ & 19 \\
Unknown IA & 4.68 & 0.73 & 29.84 & 0.1 & 41 \\
IA:tubal factor & 12.95 & 1.28 & 131.11 & $0.03 *$ & 11 \\
IA:ovulatory disorder & 10.84 & 1.47 & 80.03 & $0.02^{*}$ & 17 \\
OR & 0.89 & 0.66 & 1.2 & 0.44 & Unknown \\
EF & 1.11 & 0.77 & 1.61 & 0.57 & Unknown \\
ET & 1.17 & 0.68 & 2.03 & 0.57 & 343 \\
\hline
\end{tabular}

\section{Discussion}

Our findings indicate that infection with CagA strains of $H$. pylori is associated with EPL following ICSI. The likelihood of EPL also increases with tubal factor and ovulatory disorder as the underlying infertility etiology. The pregnancy rates were not affected by $H$. pylori infection nor CagA positivity, but were affected by maternal age $(P=0.0001)$.

We also observed a statistically significant link between the increase in age and $H$. pylori infection in infertile women $(P=0.040)$, which has not been reported previously.

This finding may indeed reflect the trend described previously in children, general population couples, and Welsh men, ${ }^{19}$ which suggests that the prevalence of $H$. pylori infection generally increases with age. ${ }^{20}$ Since age has been reported to contribute to the increase of EPL after ICSI, we may hypothesize that undetected CagA-positive $H$. pylori infection among subjects in those studies may have been an additional factor underlying this observed trend. We observed no association between EPL and maternal age $(P=0.15)$.

Although ICSI is an efficient solution for many infertile couples, it is generally accompanied by high rates of EPL, ranging from $12 \%$ to $48 \% .{ }^{21}$ EPL not only significantly reduces the efficiency of the treatment, it also increases the psychological burden on patients undergoing the procedure. ${ }^{21}$

Various factors have been comprehensively reported to contribute to EPL in ICSI, including overweight, ${ }^{22}$ ovarian stimulation with GnRH antagonists, ${ }^{23}$ maternal age, ${ }^{9}$ smoking, and the transfer of poor quality embryos. ${ }^{21}$ Based on our data, we postulate that other causes of EPL following ICSI include infection with $H$. pylori CagA-positive strains and tubal factor and ovulation disorder.

Recently the evidence indicating the causative role of $H$. pylori infection in pregnancy failure has been rapidly accumulating: In males, it has been shown that
H. pylori infection may affect semen quality in men with idiopathic infertility ${ }^{14}$ with two hypotheses explaining this finding: inflammatory reaction to infection, and the immune reaction between $H$. pylori and the sperm antigen.

It has been demonstrated that CagA-positive strains of H. pylori can cause injury to different organ systems including male and female reproductive organs through the release of different inflammatory mediators, such as IL-8, IL-1 $\beta$, and TNF- $\alpha .{ }^{24} \mathrm{TNF}-\alpha$ is multifunctional cytokine with a regulatory role in many fundamental processes, such as immunologic response, angiogenesis, or apoptosis, and is formed both by the placenta and the uterus. It is also associated with apoptosis in different cell lines including the sperm, and has a negative effect on sperm motility. Increase in TNF- $\alpha$ production can lead to pregnancy failure, ${ }^{25}$ and TNF- $\alpha$ levels are reportedly high in patients infected with CagA-positive H. pylori strains. ${ }^{14,26}$ It may be assumed that CagA-positive strains of $H$. pylori could increase the rates of EPL through TNF- $\alpha$-related mechanisms.

Our study has detected a significant correlation between different infertility causes and EPL, specifically ovarian dysfunction and tubal factor. This is the first study to observe such a trend.

Polycystic ovary syndrome (PCOS) has been linked to an increased risk of miscarriage, ${ }^{27}$ but exact mechanisms related to this observance remain enigmatic. Various factors have been implicated, including dysfunction of the Dicer, ${ }^{29}$ angiotensin-converting enzyme and plasminogen activator inhibitor-1 gene polymorphisms, ${ }^{30}$ and interestingly, PCOS has been linked to H. pylori infection. ${ }^{31}$ Although our data do not provide sufficient insight into the exact mechanism underpinning EPL in women with ovarian dysfunction infertility, they demonstrate with high significance that this infertility etiology is an independent risk factor for EPL.

Tubal infertility and EPL has been previously linked to total antioxidant capacity, ${ }^{27}$ otherwise there is no evidence 
linking this infertility etiology to EPL, nor there is an evidence linking tubal infertility with $H$. pylori.

The CagA seropositivity in Iran has been reported to reach $72.8 \%{ }^{28}$ Infection with $H$. pylori CagA-positive strains has been shown to cause a severe inflammatory response and significant neutrophil infiltration in the gastric mucosa. ${ }^{29}$ Our finding of a statistically significant relationship between CagA-positive strains of $H$. pylori and EPL might be explicable on the basis of general inflammatory reaction to infection. Concentrations of IL- $1 \beta$, IL- 8 , and TNF- $\alpha$ were all significantly higher in $H$. pylori-positive gastric mucosa samples. ${ }^{30}$ These cytokines may cause systemic inflammation that could affect the integrity of the fetoplacental unit and threaten the welfare of the foetus. ${ }^{31}$ It was shown that poor oral hygiene is associated with a history of miscarriage, ${ }^{31}$ possibly due to systemic inflammatory response to oral bacterial infection. It may be assumed that a parallel mechanism underpins the EPL in women infected with CagA-positive H. pylori strains.

The limitations of our study include the determination of $H$. pylori infection with only one method; although the ELISA kit employed in this study has sensitivity and specificity of about $85 \%$. Additionally, the number of women with CagA-positive strain $H$. pylori infection was relatively low. We also included no paternal variable in the study.

Based on our results, we hypothesize that CagA-positive H. pylori strains play a significant role in EPL after ICSI. Since ICSI is generally a very straining, time-consuming, and costly procedure, and the eradication of $H$. pylori is relatively cheap and easy to achieve, we propose that CagA status should be determined in all patients undergoing ICSI, and the infection should be treated in positive patients prior to ICSI.

We also propose a large-scale study to be conducted on the effect of $H$. pylori infection on implantation and EPL in pregnancies conceived naturally, to further elucidate the role of $H$. pylori in pregnancy failure. Further studies are also required to elucidate the role of specific etiologic factors of infertility in EPL and related mechanisms.

\section{Disclosure}

The authors report no conflicts of interest in this work.

\section{References}

1. Hajishafiha M, Zobairi T, Zanjani VR, Ghasemi-Rad M, Yekta Z, Mladkova N. Diagnostic value of sonohysterography in the determination of fallopian tube patency as an initial step of routine infertility assessment. J Ultrasound Med. 2009;28(12):1671-1677.

2. Templeton A. Infertility and the establishment of pregnancy - overview. Br Med Bull. 2000;56(3):577-587.
3. Smith S, Pfeifer SM, Collins JA. Diagnosis and management of female infertility. JAMA. 2003;290(13):1767-1770.

4. Cox GF, Burger J, Lip V, et al. Intracytoplasmic sperm injection may increase the risk of imprinting defects. Am J Hum Genet. 2002;71(1):162-164

5. Human Fertilisation and Embryology Authority, SAID. Latest UK IVF figures 2007 cited 2007; Available from: http://www.hfea.gov.uk/ ivf-figures-2006.html\#1283. Accessed on September 13, 2011.

6. Van Steirteghem AC, Nagy Z, Joris H, Liu J, et al. High fertilization and implantation rates after intracytoplasmic sperm injection. Hum Reprod. 1993;8(7):1061-1066.

7. Goker EN, Sendag F, Levi R, Sendag H, Tavmergen E. Comparison of the ICSI outcome of ejaculated sperm with normal, abnormal parameters and testicular sperm. Eur J Obstet Gynecol Reprod Biol. 2002;104(2):129-136.

8. van Golde RJ, Wetzels AM, de Graaf R, Tuerlings JH, Braat DD, Kremer JA. Decreased fertilization rate and embryo quality after ICSI in oligozoospermic men with microdeletions in the azoospermia factor c region of the Y chromosome. Hum Reprod. 2001;16(2): 289-292.

9. Bahceci M, Ulug U. Does underlying infertility aetiology impact on first trimester miscarriage rate following ICSI? A preliminary report from 1244 singleton gestations. Hum Reprod. 2005;20(3):717-721.

10. Fedorcsak P, Storeng R, Dale PO, Tanbo T, Abyholm T. Obesity is a risk factor for early pregnancy loss after IVF or ICSI. Acta Obstet Gynecol Scand. 2000;79(1):43-48.

11. Hamlet A, Thoreson AC, Nilsson O, Svennerholm AM, Olbe L. Duodenal Helicobacter pylori infection differs in cagA genotype between asymptomatic subjects and patients with duodenal ulcers. Gastroenterology. 1999;116(2):259-268.

12. Redeen S, Petersson F, Tornkrantz E, Levander H, Mardh E, Borch K. Reliability of diagnostic tests for Helicobacter pylori infection. Gastroenterol Res Pract. 2011;2011:940650.

13. Figura N, Piomboni P, Ponzetto A, et al. Helicobacter pylori infection and infertility. Eur J Gastroenterol Hepatol. 2002;14(6):663-669.

14. Collodel G, Moretti E, Campagna MS, Capitani S, Lenzi C, Figura N. Infection by CagA-positive Helicobacter pylori strains may contribute to alter the sperm quality of men with fertility disorders and increase the systemic levels of TNF-alpha. Dig Dis Sci. 2010;55(1): 94-100.

15. Rossi G, Romagnoli S, Lauretti L, et al. Helicobacter pylori infection negatively influences pregnancy outcome in a mouse model. Helicobacter. 2004;9(2):152-157.

16. Yamaoka Y, Kodama T, Gutierrez O, Kim JG, Kashima K, Graham DY. Relationship between Helicobacter pylori iceA, cagA, and vacA status and clinical outcome: studies in four different countries. $J$ Clin Microbiol. 1999;37(7):2274-2279.

17. Graham DY, Yamaoka Y. H. pylori and cagA: relationships with gastric cancer, duodenal ulcer, and reflux esophagitis and its complications. Helicobacter. 1998;3(3):145-151.

18. Aittomaki K, Wennerholm UB, Bergh C, Selbing A, Hazekamp J, Nygren KG. Safety issues in assisted reproduction technology: should ICSI patients have genetic testing before treatment? A practical proposition to help patient information. Hum Reprod. 2004;19(3): 472-476.

19. Sitas F, Forman D, Yarnell JW, et al. Helicobacter pylori infection rates in relation to age and social class in a population of Welsh men. Gut. 1991;32(1):25-28.

20. Malaty HM, El-Kasabany A, Graham DY, et al. Age at acquisition of Helicobacter pylori infection: a follow-up study from infancy to adulthood. Lancet. 2002;359(9310):931-935.

21. Winter E, Wang J, Davies MJ, Norman R. Early pregnancy loss following assisted reproductive technology treatment. Hum Reprod. 2002;17(12):3220-3223.

22. Fedorcsak P, Dale PO, Storeng R, et al. Impact of overweight and underweight on assisted reproduction treatment. Hum Reprod. 2004;19(11):2523-2528. 
23. Bahceci M, Ulug U, Sismanoglu A, Tosun S, Cengiz B. Early pregnancy loss rates were different among singleton gestations conceived by ICSI using GnRH agonist and antagonist. J Assist Reprod Genet. 2009;26(4):227-229.

24. Dufour C, Brisigotti M, Fabretti G, Luxardo P, Mori PG, Barabino A. Helicobacter pylori gastric infection and sideropenic refractory anemia. J Pediatr Gastroenterol Nutr. 1993;17(2):225-227.

25. Eslick GD, Yan P, Xia HH, Murray H, Spurrett B, Talley NJ. Foetal intrauterine growth restrictions with Helicobacter pylori infection. Aliment Pharmacol Ther. 2002;16(9):1677-1682.

26. Arslan E, Colakoglu M, Celik C, et al. Serum TNF-alpha, IL-6, lupus anticoagulant and anticardiolipin antibody in women with and without a past history of recurrent miscarriage. Arch Gynecol Obstet. 2004;270(4):227-229.
27. Hart R. PCOS and infertility. Panminerva Med. 2008;50(4):305-314.

28. Jafarzadeh A, Rezayati MT, Nemati M. Specific serum immunoglobulin $\mathrm{G}$ to $\mathrm{H}$. pylori and CagA in healthy children and adults (south-east of Iran). World J Gastroenterol. 2007;13(22):3117-3121.

29. Segal ED, Cha J, Lo J, Falkow S, Tompkins LS. Altered states: involvement of phosphorylated CagA in the induction of host cellular growth changes by Helicobacter pylori. Proc Natl Acad Sci U S A. 1999;96(25):14559-14564.

30. Guiraldes E, Duarte I, Pena A, et al. Proinflammatory cytokine expression in gastric tissue from children with Helicobacter pylori-associated gastritis. J Pediatr Gastroenterol Nutr. 2001;33(2):127-132.

31. Heimonen A, Janket SJ, Meurman JH, Furuholm J, Ackerson LK, Kaaja R. Oral health care patterns and the history of miscarriage. Oral Dis. 2008;14(8):734-740.
International Journal of Women's Health

\section{Publish your work in this journal}

The International Journal of Women's Health is an international, peerreviewed open-access journal publishing original research, reports, reviews and commentaries on all aspects of women's healthcare including gynecology, obstetrics, and breast cancer. Subject areas include: Chronic conditions (migraine headaches, arthritis, osteoporosis);

\section{Dovepress}

Endocrine and autoimmune syndromes; Sexual and reproductive health; Psychological and psychosocial conditions. The manuscript management system is completely online and includes a very quick and fair peer-review system. Visit http://www.dovepress.com/ testimonials.php to read real quotes from published authors.

Submit your manuscript here: http://www.dovepress.com/international-journal-of-womens-health-journal 